\title{
JOGOS ELETRÔNICOS NA REABILITAÇÃO DO EQUILÍBRIO CORPORAL EM IDOSO COM DOENÇA VESTIBULAR: CASO CLÍNICO
}

\author{
Flávia DONÁ ${ }^{1}$ \\ Janete Pereira de Faria ARAÚJO² \\ Denise Alves dos Reis MAIA ${ }^{3}$ \\ Adirléia Machado ALVES ${ }^{4}$ \\ Cristiane Akemi KASSE ${ }^{4}$
}

1. Fisioterapeuta, doutora pela Universidade Federal de São Paulo (UNIFESP) e docente do Programa de Mestrado Profissional em Reabilitação do Equilíbrio Corporal e Inclusão Social da Universidade Anhanguera de São Paulo.

2. Fisioterapeuta, graduada pela Universidade Anhanguera de São Paulo.

3. Fisioterapeuta, aluna do Programa de Mestrado Profissional em Reabilitação do Equilíbrio Corporal e Inclusão Social da Universidade Anhanguera de São Paulo.

4. Fisioterapeuta, especialista pela Universidade Estadual de Campinas (UNICAMP) e aluna do Programa de Mestrado Profissional em Reabilitação do Equilíbrio Corporal e Inclusão Social da Universidade Anhanguera de São Paulo.

5. Otorrinolaringologista, doutora pela Universidade Federal de São Paulo (UNIFESP) e docente do Programa de Mestrado Profissional em Reabilitação do Equilíbrio Corporal e Inclusão Social da Universidade Anhanguera de São Paulo.

Corresponding author: Flávia Doná, PT, PhD.

Endereço: Rua Maria Cândida, 1.813, 02071-013, São Paulo - SP, Brazil. Programa de Mestrado em Reabilitação do Equilíbrio Corporal e Inclusão Social da Universidade Anhanguera de São Paulo. E-mail: flavia.dona@gmail.com

\section{Recebido em: 30/05/2014 - Aprovado em: 30/06/2014 - Disponibilizado em: 30/07/2014}

RESUMO: O objetivo deste estudo foi avaliar os efeitos da reabilitação do equilíbrio corporal (RE), por meio do ambiente de realidade virtual Nintendo Wii ${ }^{\mathrm{TM}}$ em uma idosa com disfunção vestibular crônica em relação à avaliação clínica e funcional. Trata-se de um relato de caso de H.F.A., do sexo feminino, 62 anos, com queixa de desequilíbrio corporal, vertigem, zumbido e dor de cabeça há cinco anos. Já havia sido tratada com medicamentos supressores, mas não obteve sucesso. A RE consistiu em doze sessões com jogos do Wii Fit Plus, duas vezes por semana, com duração de 60 minutos cada sessão. Como métodos de avaliação foram aplicados o Questionário Funcional Multidimensional Brasileiro (BOMFAQ), Dizziness Handicap Inventory (DHI), Dynamic Gait Index (DGI) e posturografia computadorizada (Balance Rehabilitation Unit). Foram escolhidos dez jogos do Wii Fit Plus e Balance Board. Após o tratamento, a paciente apresentou remissão da tontura e redução da intensidade e frequência do zumbido e da dor de cabeça. Houve melhora na capacidade funcional, qualidade de vida (DHI antes RE = 22 pontos; após RE $=02$ pontos) e estabilidade dinâmica (DGI antes $\mathrm{RE}=18$ pontos; após $\mathrm{RE}=24$ pontos), aumento da área do limite de estabilidade (antes $\mathrm{RE}=52 \mathrm{~cm}^{2}$; após $\mathrm{RE}=171 \mathrm{~cm}^{2}$ ) e redução da oscilação corporal em diferentes condições sensoriais. A reabilitação por meio da tecnologia dos jogos Wii ${ }^{\mathrm{TM}}$ Fit Plus foi bem sucedida na promoção da melhora clínica e funcional de uma paciente com distúrbio do equilíbrio corporal de origem vestibular.

Palavras chave: Equilíbrio. Vertigem. Idoso. Reabilitação. Labirinto.

\section{ELECTRONIC GAMES FOR BALANCE REHABILITATION IN ELDERLY PEOPLE WITH VESTIBULAR DISORDER: CASE REPORT}

\begin{abstract}
The aim of this study was to evaluate the effects of balance rehabilitation (BR) using a virtual reality environment Nintendo Wii ${ }^{\mathrm{TM}}$ in an elderly with chronic vestibular dysfunction and relationship to clinical and functional assessment. This is a case report of HFA, female, 62 years old, complaining of imbalance, vertigo, tinnitus, and headache for five years. Had already been treated with suppressive medication, but was unsuccessful. BR consisted of twelve sessions with the Wii Fit Plus games held twice a week, lasting 60 minutes each session. As methods for evaluating the Brazilian Multidimensional Functional Questionnaire (BOMFAQ), Dizziness Handicap Inventory (DHI), Dynamic Gait Index (DGI) and computerized posturography (Balance Rehabilitation Unit) were performed. Ten games for Wii Fit Balance Board were chosen. After treatment, the patient showed a remission of dizziness and a reduction in the intensity and frequency of tinnitus and headache. There was an improvement in functional capacity, quality of life $(\mathrm{DHI} B R=22$ points before and after $\mathrm{BR}=02$ points), the gait performance $(\mathrm{DGI} \mathrm{BR}=18$ points before and after $\mathrm{BR}=$ 24 points), increased limit of stability ( $B R=52 \mathrm{~cm}^{2}$ before and after $B R=171 \mathrm{~cm}^{2}$ ) and a reduction of body sway in differents sensorial conditions. The rehabilitation by Wii ${ }^{\mathrm{TM}}$ Fit Plus game technology has been successful in promoting clinical and functional improvement in a patient with vestibular balance disorder.
\end{abstract}

Key words: Balance. Vertigo. Elderly. Rehabilitation. Labyrinth. 


\section{INTRODUÇÃO}

O equilíbrio corporal é a capacidade do indivíduo de manter-se ereto ou executar movimentos de aceleração e rotação do corpo, sem instabilidade postural ou quedas, de maneira harmônica e sincronizada. Esta habilidade depende da integração de informações sensoriais (visuais, somatossensoriais e vestibulares) e motoras (HORAK, 2006).

A disfunção vestibular assume particular importância durante o envelhecimento devido à deterioração funcional do labirinto. $\mathrm{O}$ paciente pode apresentar vários sintomas concomitantes, tais como, tontura, perda auditiva, zumbido, instabilidade postural, distúrbios na marcha, fraqueza nos membros inferiores, incapacidades e histórico de quedas recorrentes (GANANÇA et al., 2008).

Os relatos de desequilíbrio corporal, desvio da marcha, instabilidade postural, náuseas, tontura e quedas são frequentes em idosos acima dos 65 anos, e estão comumente associados a diversas doenças e/ou ao uso de múltiplos medicamentos ou seus efeitos colaterais. O desequilíbrio corporal causa grandes limitações nas atividades de vida diária e sociais do idoso, pois a instabilidade postural gera insegurança física e psíquica, ansiedade, isolamento social, depressão e pânico.

As vestibulopatias prevalentes na população idosa são: vertigem posicional paroxística benigna, neurite vestibular, doença de Ménière, labirintopatias metabólicas, traumas, insuficiência vertebrobasilar, tumores, ototoxicoses etc. (GANANÇA et al., 2008). Além disso, o próprio envelhecimento compromete a capacidade de processamento dos sinais vestibulares e das habilidades do sistema nervoso central, somada à redução da acuidade visual e somatossensorial, com diminuição da capacidade de modificações dos reflexos adaptativos e proativos, acompanhada de diversas alterações biomecânicas.

As principais formas de tratamento para as disfunções vestibulares são a medicamentosa e/ou a Reabilitação Vestibular (RV). A RV procura restabelecer o equilíbrio corporal estimulando e acelerando os mecanismos naturais de neuroplasticidade no sistema nervoso central por meio de exercícios específicos dos olhos, cabeça e/ou corpo com o objetivo de corrigir ou suprimir as informações sensoriais alteradas ou ausentes (HERDMAN, 2002; HORAK et al., 2009).

A RV quando feita de forma personalizada, a qual considera as queixas do paciente, o quadro clínico e os achados do exame vestibular, tem resultados melhores em relação à RV generalizada, com melhora em $85 \%$ dos indivíduos (GANANÇA; GANANÇA, 2001). Estudo de revisão sistemática, sobre RV em adultos de meia idade e idosos, identificou cinco pesquisas randomizadas compostas somente por idosos 
acima de 60 anos de idade. Os trabalhos mostraram que exercícios de RV convencional podem reduzir a tontura, o impacto da tontura na qualidade de vida, melhorar o equilíbrio corporal, a marcha, a capacidade funcional e reduzir o risco de quedas (RICCI et al., 2010).

O console Nintendo Wii ${ }^{\mathrm{TM}}$ é uma das plataformas de realidade virtual não imersiva mais utilizadas atualmente na reabilitação físico-funcional (LOUREIRO et al., 2012; MUSSATO et al., 2012; BIERYLA; DOLT, 2013; PAVÂO et al., 2013;). É de baixo custo e fácil acesso no tratamento de pacientes com doenças neurológicas. $\mathrm{O}$ trabalho multissensorial promovido pelo videogame permite a uma pessoa receber, interpretar e integrar estímulos para ser capaz de interagir com o ambiente e outras pessoas.

Embora utilizados na prática clínica, não se observou evidências científicas da aplicação de jogos eletrônicos na reabilitação do equilíbrio corporal de idosos com disfunção vestibular crônica. A finalidade de empregar jogos eletrônicos na reabilitação de pacientes com distúrbio do equilíbrio corporal de origem vestibular é recriar mudanças ambientais dos estímulos visuais, auditivos, vestibulares e somatossensoriais para ajustar os reflexos vestíbulo-ocular e vestíbuloespinhal, envolvidos no controle postural e nas estratégias reativas e proativas do equilíbrio corporal. Este estudo objetivou avaliar os efeitos dos Jogos Interativos do Nintendo Wii ${ }^{\mathrm{TM}}$ no equilíbrio corporal de uma idosa com disfunção vestibular de origem periférica.

\section{MÉTODO}

Trata-se de estudo prospectivo de um caso clínico atendido no Laboratório de Estudo e Pesquisa em Reabilitação do Equilíbrio Corporal e Inclusão Social da Universidade Anhanguera de São Paulo/SP, conduzido dentro dos princípios éticos de acordo com a Resolução 466/12 do Conselho Nacional de Saúde e aprovado pelo Comitê de Ética em Pesquisa Envolvendo Seres Humanos da Instituição (0006/08). A paciente participou voluntariamente, concordou e assinou o Termo de Consentimento Livre e Esclarecido.

O tratamento foi realizado duas vezes por semana, totalizando 12 sessões de 60 minutos. Os testes para avaliar o resultado do tratamento antes de depois da RV foram os dados clínicos, o questionário Brazilian OARS Multidimensional Functional Assessment Questionnaire (BOMFAQ) (RAMOS et al., 1993), o Dizziness Handicap Inventory (DHI) (CASTRO et al., 2007), o Dynamic Gait Index (DGI) (CASTRO; PERRACINI; GANANÇA, 2006) e o limite de estabilidade (LE), área de oscilação do centro de pressão do corpo (COP) e a velocidade de oscilação (VOC) por meio da posturografia estática do Balance Rehabilitation Unit - BRUT' $\left(B R U^{\circledR}\right.$, 2006). Os dados clínicos avaliados foram números de doenças, hipótese diagnóstica 
sindrômica e topográfica da disfunção vestibular, classificação da disfunção vestibular quanto ao tipo de afecção, tempo de início da tontura, tipo de tontura, duração da tontura, periodicidade da tontura e sintomas associados.

O BOMFAQ avalia a dificuldade referida na realização de 15 atividades de vida diária (AVD), sendo oito atividades físicas de vida diária (AFVD) e sete atividades instrumentais de vida diária (AIVD). Foi quantificado o número de AVD referidas como difíceis para realizar, totalizando no final o número de atividades comprometidas. A dificuldade em desempenhar as tarefas cotidianas, quando presente, é categorizada em "muita" e "pouca", porém neste estudo foi considerada apenas a presença ou não de dificuldade na atividade referida.

O DHI versão brasileira avalia a interferência da tontura na qualidade de vida. Apresenta 25 questões (07 referentes ao aspecto físico, 09 ao aspecto emocional e 09 ao aspecto funcional). Cada resposta "sim" do paciente vale 04 pontos, "às vezes", 02 pontos, e a resposta "não", nenhum ponto. A sua pontuação varia de 0 a 100 pontos, sendo que, quanto mais próximo do valor máximo 100, maior será a desvantagem causada pela tontura na vida do paciente.

O DGI é composto por oito tarefas de deambulação: 1- velocidade e instabilidade da marcha desempenhada pelo indivíduo em sua velocidade normal, 2- aceleração e desaceleração, 3- movimento de rotação cefálica, 4- movimento de flexo-extensão cefálica, 5- movimento de rotação axial do corpo, 6- ultrapassagem de obstáculo (caixa de sapato), 7- circundar obstáculo (cones de trânsito) e 8- subir e descer escada. A pontuação é baseada em conceitos da disfunção ausente (três pontos), mínima (dois pontos), moderada (um ponto) ou acentuada (zero) da marcha, enquanto são desempenhadas as oito tarefas de deambulação. Os escores das oito tarefas são somados em um escore total que varia entre 0 a 24 pontos, sendo o maior escore relacionado a um melhor desempenho.

A posturografia por meio do $\mathrm{BRU}^{\mathrm{TM}}$ fornece informações sobre a área do LE, e a área do COP e da VOS nas seguintes condições: superfície firme e olhos abertos; superfície firme e olhos fechados; superfície instável e olhos fechados; estímulo sacádico em superfície firme; estímulos optocinéticos (barras para à direita) em superfície firme; estímulos optocinéticos (barras para à esquerda) em superfície firme; estímulos optocinéticos (barras para baixo) em superfície firme; estímulos optocinéticos (barras para cima) em superfície firme; interação visuovestibular (barras - horizontal) em superfície firme e interação visuovestibular (barras - vertical) em superfície firme (GAZZOLA et al., 2009).

No protocolo de tratamento com jogos do Nintendo Wii ${ }^{\mathrm{TM}}$ selecionaram-se 10 jogos do Wii Fit Plus, sendo a maioria associado ao Wii Balance Board. Os acessórios do 
Nintendo Wii ${ }^{\mathrm{TM}}$ utilizados foram: Wii Remote Controller, que detecta a aceleração e orientação em três dimensões, e uma plataforma de equilíbrio corporal (Wii Balance Board). Os jogos selecionados foram o Free Run (1- olhos abertos e fechados/ superfície firme; 2- movimento de rotação cefálica / superfície firme; 3- movimento de flexo-extensão de cabeça / superfície firme; 4olhos abertos e fechados / superfície instável - almofada de espuma de média densidade), Soccer Heading, Pinguin Slide, Bambolê, Island Cycling, Perfect 10, Tilt Table, Tight Rope, Free Steps (olhos abertos e fechados na superfície fixa e instável - espuma) e Balance Bubble por treinarem o equilíbrio corporal utilizando estímulos optocinético, sacádico, perseguição ocular lenta, interação visuovestibular-somatossensorial e dupla tarefa, além de treinarem os ajustes posturais anteroposteriores e látero-laterais, estratégias reativas e proativas do equilíbrio corporal. A reabilitação foi mantida por duas vezes por semana, totalizando doze sessões.

O tratamento fisioterapêutico convencional não foi mantido durante a execução deste estudo. Não orientamos exercícios para realizar no domicílio, pois poderiam interferir nos resultados finais da pesquisa. Cada uma das sessões obedeceu ao nível de adaptação da paciente aos exercícios, procurando-se sempre atingir todas as etapas do protocolo, bem como, foi observada a manifestação dos sintomas, buscando o limite do desconforto suportável para a paciente na execução de cada tarefa. A etapa de avaliação foi realizada na semana anterior ao início dos protocolos terapêuticos e também na semana posterior a última sessão, efetuada por examinadores treinados, que não participaram da intervenção terapêutica.

\section{RESULTADOS}

Paciente com 62 anos de idade, sexo feminino, com diagnóstico de disfunção vestibular periférica crônica decorrente ao envelhecimento. A queixa principal era instabilidade postural, sensação de pressão na cabeça, tontura rotatória e zumbido na orelha direita há cinco anos, sem tratamento prévio com medicação ou reabilitação. Na avaliação inicial referiu tontura de caráter rotatório há mais de cinco anos, com piora progressiva, duração de segundos e de periodicidade esporádica. A tontura era presente, principalmente, durante o movimento de cabeça. Outros sintomas otoneurológicos concomitantes a tontura estão descritos na Tabela 1.

No exame físico otorrinolaringológico, apresentou otoscopia normal, ausência de nistagmo espontâneo e semiespontâneo, teste de Dix Hallpike e Brand Daroff negativos, pares cranianos normais, provas cerebelares e teste de Romberg normais. Não apresentou anormalidade nos testes de Romberg Barré e Untemberg Fukuda. Observou-se uma marcha com leve instabilidade lateral, oscilação ântero-posterior e estratégia de passo. A 
vectonistagmografia computadorizada foi normal. Referiu dificuldade para realizar seis atividades do BOMFAQ: subir escada, medicar-se na hora, fazer compras, andar perto de casa, cortar as unhas dos pés, sair de condução e limpar a casa (Tabela 2). A pontuação no DGI foi de 18 (indicativo de maior risco de quedas), conforme mostra a Tabela 3. Na pontuação do DHI prétratamento observou-se: 08 pontos na subescala física, 08 pontos na subescala emocional e 06 pontos na subescala funcional, totalizando 22 pontos.

Tabela 1 - Manifestações clínicas antes e após as 12 sessões de reabilitação.

\begin{tabular}{|c|c|c|}
\hline Sintomas & Pré-RE & Pós-RE \\
\hline Tontura & + & - \\
\hline Hipoacusia & - & - \\
\hline Zumbido & + & $\downarrow$ \\
\hline Desequilíbrio corporal & + & - \\
\hline Oscilopsia & - & - \\
\hline Insônia & + & $\downarrow$ \\
\hline Fraqueza em MMII & + & - \\
\hline Cefaleia & + & $\downarrow$ \\
\hline Deambulação independente & + & + \\
\hline \multicolumn{3}{|c|}{$\begin{array}{l}\text { Presente (+); Ausente (-); Presente Diminuído ( } \downarrow \text { ) } \\
\text { Legenda: RE - reabilitação do equilíbrio corporal. }\end{array}$} \\
\hline \multicolumn{3}{|c|}{$\begin{array}{l}\text { Na reavaliação observou-se: remissão da } \\
\text { tontura, da sensação de desmaio iminente, } \\
\text { do distúrbio da memória/concentração, da } \\
\text { cinetose e das crises de vômitos e redução } \\
\text { de } 50 \% \text { da intensidade da cefaleia, } \\
\text { zumbido e ganho de força muscular em } \\
\text { membros inferiores (Tabela } 1 \text { ); redução da }\end{array}$} \\
\hline
\end{tabular}

$\mathrm{RE}=02$ ); melhora da capacidade funcional (Tabela 2); maior desempenho à marcha sem risco de quedas (Tabela 3); aumento da área do LE (Pré-RE=52 cm²; Pós$\mathrm{RE}=171 \mathrm{~cm}^{2}$ ) e redução da oscilação corporal (Tabela 4).

Tabela 2 - Capacidade funcional antes e após as 12 sessões de reabilitação.

\begin{tabular}{lll}
\hline BOMFAQ & Pré-RE & Pós-RE \\
\hline
\end{tabular}

Subir escada (01 lance)

Medicar-se na hora

Fazer compras

Cortar unhas dos pés

Sair de condução

Fazer limpeza de casa

Presente (+) Ausente (-)

Legenda: RE - reabilitação do equilíbrio corporal.

Tabela 3 - Pontuação no Dynamic Gait Index (DGI) antes e após as 12 sessões de reabilitação.

Tarefas

Pré- RE Pós-RE

1-Marcha em superfície plana

2-Mudança na velocidade da

marcha

$3 \quad 3$

3-Marcha com rotação

horizontal de cabeça $\quad 2 \quad 3$

4-Marcha com rotação vertical de cabeça

$3 \quad 3$




\begin{tabular}{lcc} 
5-Marcha e rotação & 2 & 3 \\
6-Passar por cima de um & & 3 \\
obstáculo & 2 & 3 \\
7-Andar ao redor do obstáculo & 2 & 3 \\
8-Degraus & 2 & $\mathbf{1 8}$ \\
\hline TOTAL & $\mathbf{2 4}$ \\
\hline
\end{tabular}

Legenda: RE - reabilitação do equilíbrio corporal.

Tabela 4 - Área do centro de pressão (COP) antes e após as 12 sessões de reabilitação.

\begin{tabular}{lcc}
\hline Condições & Pré-RE & Pós-RE \\
\hline SF, AO & $0,89 \mathrm{~cm}^{2}$ & $0,63 \mathrm{~cm}^{2}$ \\
SF, OF & $1,79 \mathrm{~cm}^{2}$ & $0,77 \mathrm{~cm}^{2}$ \\
SE OF & $13,81 \mathrm{~cm}^{2}$ & $5,01 \mathrm{~cm}^{2}$ \\
Sacádico, SF & $0,80 \mathrm{~cm}^{2}$ & $1,70 \mathrm{~cm}^{2}$ \\
OPT, Barras $\rightarrow$, SF & $1,46 \mathrm{~cm}^{2}$ & $1,21 \mathrm{~cm}^{2}$ \\
OPT, Barras $\leftarrow$, SF & $1,15 \mathrm{~cm}^{2}$ & $0,78 \mathrm{~cm}^{2}$ \\
OPT, Barras $\downarrow$, SF & $3,02 \mathrm{~cm}^{2}$ & $0,61 \mathrm{~cm}^{2}$ \\
OPT, Barras $\uparrow$, SF & $3,31 \mathrm{~cm}^{2}$ & $0,89 \mathrm{~cm}^{2}$ \\
IVV, Circular, Barras (direção & & \\
horizontal), SF & $3,05 \mathrm{~cm}^{2}$ & $1,80 \mathrm{~cm}^{2}$ \\
IVV, Circular, Barras (direção & & \\
vertical), SF & $1,88 \mathrm{~cm}^{2}$ & $2,16 \mathrm{~cm}^{2}$ \\
\hline
\end{tabular}

Legenda: SF - superfície firme; SE - superfície espuma; OA - olhos abertos; OF - olhos fechados; $\rightarrow$ (direita); $\leftarrow$ (esquerda); $\downarrow$ (para baixo); $\uparrow$ (para cima) e IVV - interação vestíbulo-visual. RE: reabilitação do equilíbrio corporal.

\section{DISCUSSÃO}

A paciente foi encaminhada à fisioterapia com o diagnóstico de presbiataxia e presbivertigem, apresentando queixa de instabilidade postural, sensação de pressão na cabeça, tontura rotatória, zumbido na orelha direita há cinco anos e fraqueza muscular. A presbiataxia e presbivertigem são ocasionadas por processos degenerativos nas estruturas sensoriais do sistema vestibular central e periférica, que se agravam com o envelhecimento (RUWER; ROSSI; SIMON, 2005; FELIPE et al., 2008).

O aumento da idade é diretamente proporcional à presença de múltiplos sintomas otoneurológicos associados: vertigem e outras tonturas, perda auditiva, zumbido, alterações do equilíbrio corporal, distúrbios da marcha, quedas recorrentes, déficit de atenção e cognição, ansiedade, depressão etc. (GANANÇA et al., 2008), sendo consistente com a história clínica da H.F.A. Na reavaliação observou-se: remissão da tontura, da sensação de desmaio iminente, do distúrbio da memória/concentração, da cinetose e das crises de vômitos e redução de $50 \%$ da intensidade da cefaleia, zumbido e ganho de força muscular em membros inferiores (Tabela 1); redução da pontuação no DHI (Pré-RE=22; Pós-RE=02); melhora na capacidade funcional (Tabela 2); maior desempenho à marcha sem risco de quedas (Tabela 3); aumento da área do limite de estabilidade (Pré-RE=52 cm²; Pós-RE=171 $\mathrm{cm}^{2}$ ) e redução da oscilação corporal (Tabela 4).

A H.F.A antes de iniciar a fisioterapia referiu limitações para realizar algumas atividades de vida diária instrumentais e/ou físicas, devido à tontura e instabilidade postural como, por exemplo, fazer a limpeza de casa, corta as unhas dos pés, fazer compras, dificuldades para subir escadas e pegar condução. Esses achados reforçam os resultados obtidos por Aratani et al. (2006), 
que idosos vestibulopatas podem apresentar maior comprometimento da capacidade funcional em relação aos idosos da comunidade e este prejuízo dificulta a realização de tarefas físicas e instrumentais de vida diária. Em relação ao impacto da tontura na qualidade de vida, observou-se que H.F.A obteve pontuação inicial no DHI de 22 (impacto leve). A intensidade da tontura era de leve a moderada, porém a presbiataxia era mais intensa, que repercutiu negativamente na locomoção e, consequentemente, no maior risco de queda.

Os objetivos primordiais da RV são: promover a estabilização visual durante a movimentação da cabeça; melhorar a interação vestibulovisual durante a movimentação cefálica; ampliar a estabilidade estática e dinâmica nas condições que produzem informações sensoriais conflitantes; e diminuir a sensibilidade individual à movimentação cefálica (GANANÇA et al., 2013). O processo de reabilitação foi personalizado e adequado para as necessidades da paciente.

Ao término da fisioterapia, observouremissão das crises vertiginosas e melhora da autoconfiança em relação ao equilíbrio corporal. $\mathrm{Na}$ avaliação funcional, houve aumento da pontuação no DGI, melhora da capacidade funcional, redução da oscilação corporal e maior desempenho em todos os jogos aplicados. Em relação aos parâmetros avaliados na posturografia, verificou-se aumento significativo do LE que sugere maior estabilidade corporal e melhor controle da estratégia reativa de tornozelo, além da redução da oscilação corporal, isto é, maior integração sensorial e menos dependência da informação visual.

Inicialmente, a paciente relatou dificuldade na realização dos exercícios por causa da sensação de tontura e desequilíbrio corporal, mas com o passar do tempo sentiuse mais segura e independente. Esta melhora foi ratificada pelo decréscimo da pontuação total do DHI, DGI associada à melhora no BOMFAQ, indicativos de melhora na sua qualidade de vida e na capacidade física e funcional.

Os jogos de interação multissensorial abrem a perspectiva no auxílio da reabilitação dos distúrbios do equilíbrio corporal de origem vestibular, ao ativar os mecanismos de neuroplasticidade envolvidos no processo de compensação da disfunção vestibular, de forma lúdica. Estudos recentes têm mostrado a efetividades de jogos eletrônicos na reabilitação física-funcional de pacientes com paralisia cerebral, ataxia cerebelar precoce, acidente vascular cerebral e doença de Parkinson (BRUMELS et al., 2008; DEUTSCH et al., 2008; SCHIAVINATO et al., 2010; LOUREIRO et al., 2012). Este recurso tecnológico não substitui a fisioterapia convencional, porém é um recurso adicional aos outros métodos convencionais de reabilitação do equilíbrio corporal. 


\section{CONCLUSÃO}

A reabilitação vestibular por meio dos jogos interativos do console Nintendo Wii ${ }^{\mathrm{TM}}$ promoveu remissão da tontura, maior controle postural, redução do risco de queda e melhora da capacidade funcional e da qualidade de vida em uma idosa com Síndrome Vestibular Periférica.

\section{REFERÊNCIAS}

ARATANI, M. C. et al. Quais atividades diárias provocam maior dificuldade para idosos vestibulopatas crônicos? Acta Orl, São Paulo, v. 24, n. 1, p.18-24, jan./mar. 2006.

BIERYLA, K. A.; DOLT, N. M. Feasibility of Wii Fit training to improve clinical measures of balance in older adults. Clinical

Interventions in Aging, Auckland, v.8, n.1, p.775-781, 2013.

BRU®. UNIDADE DE REABILITAÇÃO DO EQUILÍBRIO. BRU®. Manual do usuário. Versão 1.0.7. Versão do Software: 1.3.5.0. Uruguai: Medicaa, 2006.

BRUMELS, K. A. et al. Comparison of efficacy between traditional and video game based balance programs. Clinical

\section{Kinesiology: Journal of the American}

Kinesiotherapy, p. 1-7. dez. 2008.
CASTRO, A. S. O. et al. Dizziness Handicap Inventory: adaptação cultural para o português brasileiro. Pró-fono, Barueri, v.19, n.1, p. 97-104, jan./abr. 2007.

CASTRO, S. M.; PERRACINI, M.R.;

GANANÇA, F.F. Versão brasileira do Dynamic Gait Index. Revista Brasileira de Otorrinolaringologia, São Paulo, v. 72, n. 6, p.817-825, nov./dec. 2006.

DEUTSCH, J. E. et al. Use of a low-cost, commercially available gaming console (Wii) for rehabilitation of an adolescent with cerebral palsy. Physical Therapy, New York, v. 88 , n. 10, p.1196-1207, oct. 2008.

FELIPE, L. et al. Presbivertigem como causa de tontura no idoso. Pró-fono, São Paulo, v. 20, n. 2, p.99-104, abr./jun. 2008.

GANANÇA, F. F. et al. Protocolos de Reabilitação Vestibular. In: ONISHI, E. T. et al. Avaliação e reabilitação do equilíbrio corporal: Abordagem interdisciplinar. São Paulo: Ektor Tsuneo Onishi, 2013. cap.10, p.133-168.

GANANÇA, M. M. et al. Como Diagnosticar e Tratar Vertigem. Revista Brasileira De Medicina, São Paulo, v. 65, n. 1, p.6-14, 2008.

GANANÇA F.F.; GANANÇA C. F.

Reabilitação vestibular: princípios e técnicas. 
In: Ganança M. M. et al. Estratégias

terapêuticas em otoneurologia. São Paulo:

Atheneu; 2001. p. 33-54.

GAZZOLA, J. M. et al. Realidade virtual na avaliação e reabilitação dos distúrbios vestibulares. Acta ORL, São Paulo, v.27, n.1, p. 22-27, 2009.

HERDMAN, S. J. Reabilitação vestibular.

São Paulo: Manole, 2002. 591 p.

HORAK, F. B. Postural Compensation for Vestibular Loss. Annals of the New York Academy of Sciences. New York, v.28, n.1, p.57-68, may. 2009.

HORAK, F. B. Postural orientation and equilibrium: what do we need to know about neural control of balance to prevent falls?

Oxford Journals, v. 35, n. 2, p. 7-11, sep. 2006.

LOUREIRO, A. P. C. et al. Feasibility of virtual therapy in rehabilitation of Parkinson's disease patients: pilot study. Fisioterapia Movimento, Curitiba, v. 25, n. 3, p. 659-666, jul./set. 2012.

MUSSATO, R; BRANDALIZE D.;

BRANDALIZE M. Nintendo Wii ${ }^{\circledR}$ e seu efeito no equilíbrio e capacidade funcional de idosos saudáveis. Revista Brasileira de Ciência e Movimento, São Paulo, v.20, n.2, p.68-75, 2012.

PAVÃO, S. L. et al. O ambiente virtual como interface na reabilitação pós-AVE: relato de caso. Fisioterapia Movimento, Curitiba, v. 6, n. 2, p.455-462, abr./jun. 2013.

RAMOS L.R. et al. Perfil do idoso em área metropolitana na região sudeste do Brasil: resultados de inquérito domiciliar. Revista de Saúde Pública, São Paulo, v.27, n.2, p.87-94, 1993.

RICCI, N.A. et al. Revisão sistemática sobre os efeitos da reabilitação vestibular em adultos de meia-idade e idosos. Revista Brasileira Fisioterapia. São Carlos, v.14, n.5, p.361-367, out./set. 2010.

RUWER, S. L.; ROSSI, A. G.; SIMON, L. F. Equilíbrio no idoso. Revista Brasileira de Otorrinolaringologia, São Paulo, v. 71, n. 3, p.298-303, maio/jun. 2005.

SCHIAVINATO, A. et al. Influência do Wii Fit no equilíbrio de paciente com disfunção cerebelar: estudo de caso. Journal of the Health Sciences Institute, São Paulo, v. 28, n. 1, p.50-52, 2010. 